\title{
ADAPTIVE MODULATION IN A SLOW RAYLEIGH FADING CHANNEL
}

\author{
J. M. Torrance, L. Hanzo \\ Dept. of Electr. and Comp. Sc., Univ. of Southampton, SO17 1BJ, UK. \\ Tel: +44-703-593 125, Fax: +44-703-594 508 \\ Email: jmt94r@ecs.soton.ac.uk, lh@ecs.soton.ac.uk \\ http://www-mobile.ecs.soton.ac.uk
}

\begin{abstract}
A novel, un-even protection Phase Shift Keying technique is proposed for the encoding of the required modulation scheme in an adaptive modem arrangement, which exhibits an improved performance in comparison to previously proposed schemes. The performance is derived numerically and a system dependent optimisation is presented. A benefit of 5 $\mathrm{dB}$ is achieved in a Rayleigh channel.
\end{abstract}

\section{INTRODUCTION}

Adaptive modulation schemes [1] vary the number of transmitted bits per symbol on a frame by frame basis. Typically a robust modulation scheme is employed to mitigate bit errors during deep fades, and a less robust scheme exhibiting a higher throughput is invoked to exploit the short term improvement of the fading envelope. The correlation between the up- and down-link channel conditions in a Time Division Duplex (TDD) scheme can be exploited to estimate the channel quality before transmission. Each frame must carry some redundant information to identify which modulation scheme has been employed; these take the form of control symbols. Failure to correctly decode the control symbols in a practical system could be catastrophic, as not only would the current frame be lost, but the data synchronisation would be jeopardized. Therefore, it is equally detrimental to over-estimate or under-estimate the number of transmitted bits per symbol.

In this treatise we proposed a novel unequal protection control symbol encoding scheme and evaluated its performance in contrast to two previously published benchmarkers. Let us initially consider the simplest possible solution, namely using a single M-ary Phase Shift Keying (PSK) symbol for transmitting the number of bits/symbol sideinformation.

\section{SINGLE PSK CONTROL SYMBOL}

A possible approach to convey the number of modulation bits/symbol side-information is to employ a single $M$-ary Phase Shift Keyed (PSK) symbol, allowing the encoding of $M$ different modulation schemes. The Symbol Error Rate (SER) of the control symbols, at a given SNR, will represent the probability of the receiver demodulating the data symbols within a frame with the wrong modulation scheme.

PIMRC'96, OCT. 1996, TAPEI, TAIWAN
The SER of an $M$-ary PSK modulation scheme in a Gaussian channel was given by Proakis [2] as:

$$
P_{M 1 g}(\gamma)=1-\int_{-\pi / M}^{\pi / M} \frac{1}{2 \pi} e^{-\gamma} \cdot I(\theta) d \theta
$$

where

$$
I(\theta)=1+\sqrt{4 \pi \gamma} \cos \theta e^{\gamma \cos ^{2} \theta} \frac{1}{\sqrt{2 \pi}} \int_{-\infty}^{\sqrt{2 \gamma} \cos \theta} e^{-x^{2} / 2} d x
$$

and $\gamma$ is the SNR. The outer integral in Equation 1 may easily be solved numerical using Romberg's method [3]. However, the inner integral is improper and may be computed using the Second Euler-Maxlaurin summation formula as described by Press et al [3]. In the case of $\gamma>40$ and $M \geq 2$ Equation 1 is approximated by:

$$
P_{M 2 g}(\gamma)=2 Q\left(\sqrt{2 \gamma} \sin \frac{\pi}{M}\right)
$$

where the Gaussian $Q(x)$ function is defined by:

$$
Q(x)=\frac{1}{\sqrt{2 \pi}} \int_{x}^{\infty} e^{-x^{2} / 2} d x .
$$

Therefore, generally Equations 1 and 3 may be written as:

$$
P_{M g}(\gamma)= \begin{cases}P_{M 1 g}(\gamma) & \text { if } S / N<40 \\ P_{M 2 g}(\gamma) & \text { otherwise }\end{cases}
$$

The above general expression for the $M$-PSK SER, which was given by Equation 5, may be used to determine the upper bound performance [4] of the $M$-PSK SER in a Rayleigh fading channel as follows:

$$
P_{M r}(S / N)=\int_{0}^{\infty} P_{M g}(s / N) \cdot F(s, S) d s,
$$

where $N$ is the noise power and $F(s, S)$ is the PDF of the fading Rician or Rayleigh channel. This PDF may be written in terms of the instantaneous received power, $s$, and the average received power $S$ as:

$$
F(s, S)=\frac{s(2+2 K)}{S} \cdot e^{-\frac{s^{2}(2+2 K)}{2 S}} \cdot e^{-K} \cdot \frac{s \sqrt{4(K+1)}}{\sqrt{S}}
$$

and

$$
F(s, S)=\frac{2 s}{S} \cdot e^{-s^{2} / S},
$$




\begin{tabular}{|l|c|c|c|c|}
\hline & $l_{1}$ & $l_{2}$ & $l_{3}$ & $l_{4}$ \\
\hline Speech Thresh. (dB) & 3.31 & 6.48 & 11.61 & 17.64 \\
Data Threshold (dB) & 7.98 & 10.42 & 16.76 & 26.33 \\
\hline
\end{tabular}

Table 1: Optimised switching levels for speech and computer data systems through a Rayleigh channel, shown in $\mathrm{dB}$

respectively, where $K$ is the Rician $K$-factor. Either of these Equations may be substituted into Equation 6. Numerical evaluation of the performance of PSK, where $M=5$, is given in Figure 1 for $K$ factors of 0,4 and 16 . These results were achieved by solving Equation 6 using the trapezium rule, with instantaneous power integration limits of $-80 \mathrm{~dB}$ and $20 \mathrm{~dB}$ and steps of $0.1 \mathrm{~dB}$; the $\mathrm{dBs}$ were relative to the mean signal level. The Figure also shows simulated performance of 5 PSK at the same $K$ values. Good correspondence between the simulated and numerical results is achieved. It can be observed that the SER reduces with increased average channel SNR and increased $K$ factor.

We have previously proposed [5] a scheme of optimising the switching levels in an adaptive modulation scheme to achieve a given target Bit Error Rate (BER) at the cost of the bits/sample (BPS) channel capacity. We optimised the switching levels in order to arrive at an upper bound performance for two schemes, one with a BER of $1.0 \times 10^{-2}$ and the other with $1.0 \times 10^{-4}$. Because of their BERs, we referred to these scehmes as speech and computer data systems and the optimised switching levels are shown in Table 1 , where the thresholds $l_{1}-l_{4}$ correspond to switching from disabling tranmissions in the lowest SNR region to binary PSK (BPSK), quaternary PSK (QPSK), 16-level Quadrature Amplitude Modulation (16QAM) and 64QAM, respectively. We note however that the associated delay or latency ramifications are currently under investigation. The acceptable SERs of the control symbols for the speech and computer data schemes are approximately $1 \times 10^{-2}$ and $1 \times 10^{-4}$, respectively. The acceptable SER may only be approximated because the dependence between control symbol errors and BER in the data symbols has not been exactly quantified. It can be seen from the Figure that in a Rayleigh channel $(K=0)$ a SER of $1 \times 10^{-2}$ and $1 \times 10^{-4}$ are achieved at average channel SNRs of approximately 20 and $40 \mathrm{dBs}$, respectively. Let us now consider the improvements achieved by majority voting.

\section{MAJORITY DECISION BASED CONTROL}

Steele and Webb [1] proposed an adaptive modulation scheme that employed one of four fixed differential modulation schemes depending upon the instantaneous channel conditions. It was proposed to use Differential PSK (DPSK) control symbols. In their system three identical differential control symbols were transmitted and a majority decision was carried out on the basis of the decoded control symbols. This scheme has been modified to use coherent detection and non-differentially encoded symbols. Irrespective of whether differential or non-differential encoding are used, this voting scheme can be be described by a Binomial distribution. Upon exploiting this binomial relationship as well as Equation 6, for non-differential transmissions over Rayleigh

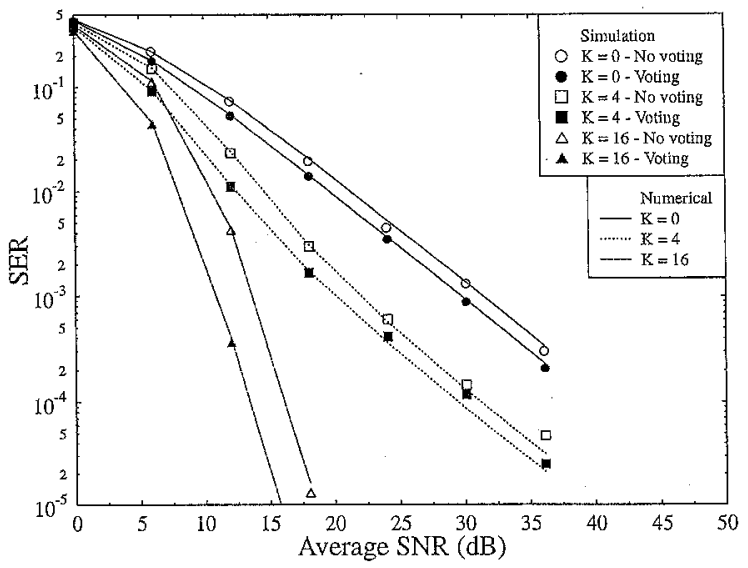

Figure 1: Simulated and numerical upper bound performance of 5 symbol PSK in fading channels with varying $K$ factors and both with 3 -symbol majority voting disabled and enabled

channels we have a control SER of:

$$
P_{M r b}(S / N)=\int_{0}^{\infty} P_{M g b}(s / N) \cdot F(s, S) d s
$$

where

$P_{M g b}(s / N)=\sum_{n=0}^{n=1}\left(\begin{array}{c}3 \\ n\end{array}\right)\left(P_{M g}(s / N)\right)^{n}\left(1-P_{M g}(s / N)\right)^{3-n}$

and $P_{M r b}(S / N)$ is the upper bound control SER of threesymbol majority decision based $M$-PSK in a Rayleigh channel.

Steele ans Webb [1] had proposed an adaptive modulation scheme that conld transmit with one of any four fixed modulation schemes and therefore the control symbols were quantized to four levels, that is $M=4$. The adaptive modulation scheme considered here employs one of five fixed modulation schemes and therefore $M=5$. Figure 1 shows the numerical solution of Equation 9 for Rician $K$ factors of 0,4 and 16 and $M=5$. Simulated results are also shown and these correspond well to the numerical results. The improvements that result from majority voting over the single control symbol transmission are approximately $1.6 \mathrm{~dB}, 2.1$ $\mathrm{dB}$ and $2.7 \mathrm{~dB}$ for $K=0, K=4$ and $K=16$ channels respectively. The explanation for the increase in differential between the majority voting and single control symbol transmission as $K$ increases is the reduction of the fade depth. This is because the channel errors now become more random and independent, rather than bursty, thereby reducing the chances of several consecutive erronous symbols. The drawback of the proposed majority voting scheme is that it adds more redundancy to the transmitted burst.

\section{DISCRETE WALSH CODES}

Otsuki et al [6] also proposed using control symbols to identify to the receiver, which modulation scheme had been employed for the data symbols. They proposed using a four 


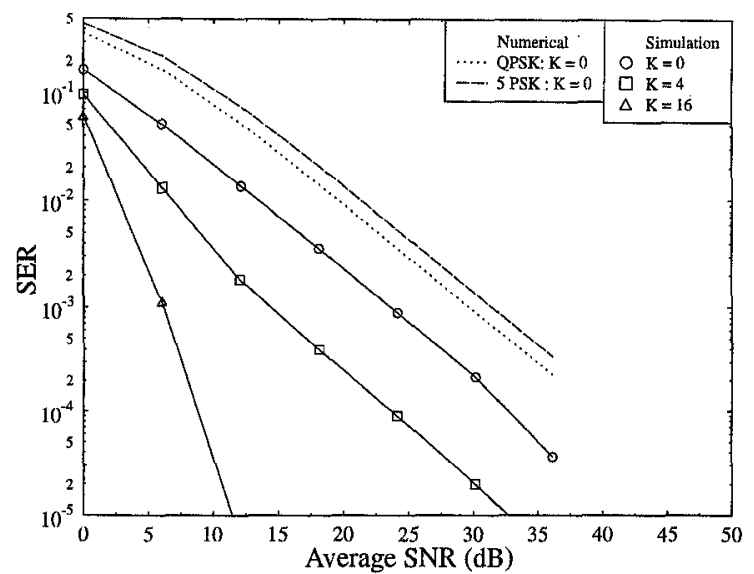

Figure 2: Simulated and numerical upper bound performance of, 4 and 5 PSK in a Rayleigh fading channel, as well as simulated performance of 4 symbol duration, 2 code Walsh control sequences in fading channels with $K$ values of 0,4 and 16

symbol Walsh code and to use maximum likelihood correlation detection to decode the Walsh codes. They suggested using simple orthogonal codes and transmitting them using BPSK, exploiting the maximum amplitude displacement in the $1^{\text {st }}$ and $3^{\text {rd }}$ Euclidean quadrants of the coordinate system. When Walsh codes of length $n$ are used, there should be $2^{n}[7](n=1,2, \ldots)$ fixed modulation schemes in order to avoid redundant mapping of the side-information on to the control symbols. A Walsh code that has $2^{n}$ levels requires at least $n \cdot T$ seconds of the frame for transmission of the code, where $T$ is the symbol duration. Otsuki et al [6] proposed an adaptive modulation scheme that could transmit with one of any four fixed modulation schemes (QPSK, 16, 64 and 256 Square QAM) and therefore the minimum codelength was $n=2$, although they opted for using $n=4$. This required using the duration of four symbols to transmit the code. The performance of the Walsh codes, as control symbols, was simulated in channels with Rician $K$ factors of 0,4 and 16. As a comparison, the performance of a single 4 PSK control symbol through a Rayleigh channel was calculated numerically from Equation 6. Both the Walsh function and the single 4 PSK control symbol performance are plotted in Figure 2. This figure also re-plots the performance of a single 5 PSK control symbol from Figure 1.

As expected, the single QPSK control symbol is slightly more robust than the single 5 PSK control symbol. The difference is about $1.5 \mathrm{~dB}$ under Rayleigh channel conditions. The Walsh code in a Rayleigh channel appears to be considerable better than the QPSK symbol, although they both signal the same information. However, the Walsh function occupies four symbol spaces. Gfeller [8] states that each time the number of samples of the same symbol is doubled and the resulting received signals averaged, the effective received SNR increases by $10 \log _{10}(2) \approx 3 \mathrm{~dB}$. This would suggest that transmitting the same single QPSK control symbol four times would result in approximately $6 \mathrm{~dB}$ SNR improvement over the single symbol performance given in Figure 2. Therefore, the error rate of a burst of four identical QPSK

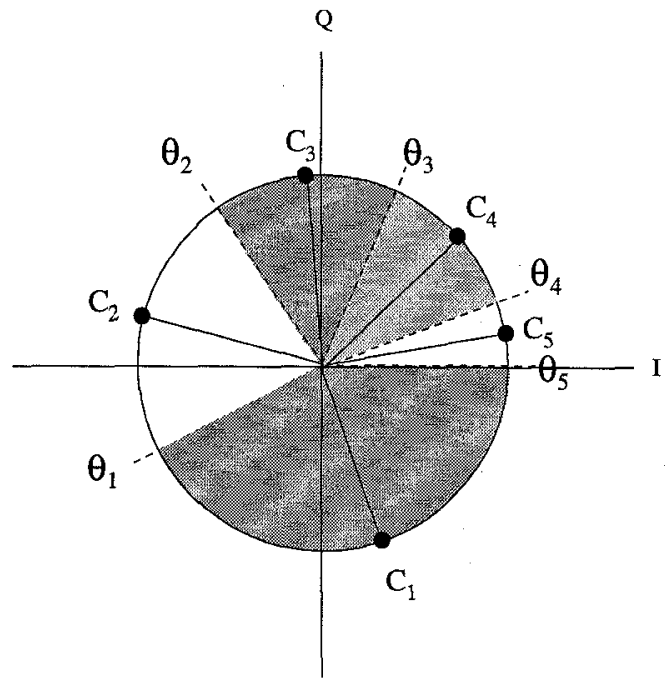

Figure 3: An uneven error protection 5 PSK symbol with complex symbol phasers at $C_{1}, C_{2} \ldots C_{5}$, and decision boundaries at $\theta_{1}, \theta_{2} \ldots \theta_{5}$

control symbols, averaged at the receiver, would be approximately coincident in Figure 2 with that of the Walsh code. For the case of four fixed modulation schemes using Walsh codes and averaging an equivalent number of times repeated QPSK symbol in order to convey the side-information have an equivalent performance. However, when the number of fixed modulation schemes deviates form $2^{n}$, the additional flexibility of the repeated PSK symbols is a more attractive than using the Walsh code for transmitting control information.

\section{UNEVEN ERROR PROTECTION}

A novel approach to the problem of control symbol transmission is to use modulation symbols that have uneven error protection. Consider a coherent PSK modulation constellation with $N$ complex vectors, $C_{1} \ldots C_{N}$, and $N$ decision boundaries at $\theta_{1} \ldots \theta_{N}$. If such a constellation was to be transmitted using QPSK then conventional thinking would maximise the minimum distance between each of the $\mathrm{N}$ vectors. This maximisation would result in the vectors being equally distributed around a circle and the decision boundaries also spaced evenly with the same separation as the constellation points. This is the case for conventional PSK because there is no correlation between the symbol transmitted and the channel conditions. However, when PSK symbols are used to transmit control information about the fixed modulation scheme that has been employed for the data in the frame, there will be a strong correlation between the channel conditions and the symbol transmitted. It is therefore proposed to use a robust symbol communicate that there is no data in the frame and accept a more noise sensitive symbol when the data is encoded with square 64 QAM. For an adaptive modulation scheme that can employ No Transmission, BPSK, QPSK and 16 or 64 square QAM this uneven error protection 5 PSK symbol is shown in Figure 3. 


\begin{tabular}{|c|c|c|c|c|c|}
\hline $\mathrm{S} / \mathrm{N}$ & $\theta_{1}-\theta_{5}$ & $\theta_{2}-\theta_{1}$ & $\theta_{3}-\theta_{2}$ & $\theta_{4}-\theta_{3}$ & $\theta_{5}-\theta_{4}$ \\
\hline $\mathrm{dB}$ & $\times 2 \pi$ & $\times 2 \pi$ & $\times 2 \pi$ & $\times 2 \pi$ & $\times 2 \pi$ \\
\hline 10 & 1.2254 & 0.8106 & 0.6997 & 0.4060 & 0.0000 \\
15 & 1.0040 & 0.7444 & 0.6971 & 0.4560 & 0.2362 \\
20 & 0.9513 & 0.7322 & 0.7010 & 0.4798 & 0.2774 \\
25 & 0.9353 & 0.7295 & 0.7031 & 0.4847 & 0.2890 \\
30 & 0.9302 & 0.7286 & 0.7036 & 0.4866 & 0.2926 \\
35 & 0.9287 & 0.7279 & 0.7037 & 0.4875 & 0.2939 \\
40 & 0.9284 & 0.7277 & 0.7036 & 0.4877 & 0.2942 \\
\hline
\end{tabular}

Table 2: Optimised $\theta_{n}$ values, for the adaptive speech system switching levels, through a Rayleigh channel, shown in Radians

\begin{tabular}{|l|c|c|c|c|c|}
\hline $\mathrm{S} / \mathrm{N}$ & $\theta_{1}-\theta_{5}$ & $\theta_{2}-\theta_{1}$ & $\theta_{3}-\theta_{2}$ & $\theta_{4}-\theta_{3}$ & $\theta_{5}-\theta_{4}$ \\
\hline $\mathrm{dB}$ & $\times 2 \pi$ & $\times 2 \pi$ & $\times 2 \pi$ & $\times 2 \pi$ & $\times 2 \pi$ \\
\hline 10 & 1.8009 & 0.6705 & 0.5244 & 0.1459 & 0.0000 \\
15 & 1.5694 & 0.7128 & 0.5688 & 0.2906 & 0.0000 \\
20 & 1.4548 & 0.6940 & 0.5771 & 0.3228 & 0.0928 \\
25 & 1.4165 & 0.6887 & 0.5791 & 0.3325 & 0.1248 \\
30 & 1.4047 & 0.6898 & 0.5793 & 0.3349 & 0.1328 \\
35 & 1.4020 & 0.6893 & 0.5800 & 0.3359 & 0.1345 \\
40 & 1.4020 & 0.6863 & 0.5797 & 0.3364 & 0.1371 \\
\hline
\end{tabular}

Table 3: Optimised $\theta_{n}$ values, for the adaptive computer data system switching levels, through a Rayleigh channel, shown in Radians

\subsubsection{Optimisation}

Each of the transmitted phasors were restricted to be in the centre of their decision thresholds. Other than that, no constraint was placed upon the values of $C_{n}$. Equation 6 may be modified to allow for the employment of different, non-integer $M$ PSK schemes depending upon the channel conditions, yielding the SER of,

$$
P_{u}\left(\frac{S}{N}\right)=\left[\begin{array}{c}
\int_{0}^{l_{1}} P_{M=\left(\frac{\pi}{\theta_{1}-\theta_{5}}\right) g}(s / N) \cdot F(s, S) d s \\
+\int_{l_{1}}^{l_{2}} P_{M=\left(\frac{\pi}{\theta_{2}-\theta_{1}}\right) g}(s / N) \cdot F(s, S) d s \\
+\int_{l_{2}}^{l_{3}} P_{M=\left(\frac{\pi}{\theta_{3}-\theta_{2}}\right) g}(s / N) \cdot F(s, S) d s \\
+\int_{l_{3}}^{l_{4}} P_{M=\left(\frac{\pi}{\theta_{4}-\theta_{3}}\right) g}(s / N) \cdot F(s, S) d s \\
+\int_{l_{4}}^{\infty} P_{M=\left(\frac{\pi}{\theta_{5}-\theta_{4}}\right) g}(s / N) \cdot F(s, S) d s
\end{array}\right]
$$

where $P_{u}(S / N)$ is the upper bound SER in a Rayleigh channel for 5-symbol uneven protection PSK. Clearly, the values for $\theta_{n}$ are dependent upon the switching levels $l_{n}$. Considering the speech and computer data systems that were introduced above it is possible to minimsie the SER in Equation 11 by finding optimum values of $\theta_{n}$ for given ranges of average channel SNRs. This was achieved using Powell's [3] optimisation, where the cost function was $P_{u}(S / N)$ and the initial conditions were $\theta_{1}-\theta_{5}=\theta_{2}-\theta_{1}=\theta_{3}-\theta_{2}=\theta_{4}-\theta_{3}=$ $\theta_{5}-\theta_{4}=0.2 \pi$. The optimisation was conducted for average SNRs of $10,15,20,25,30,35$ and $40 \mathrm{~dB}$ and for each average channel SNR value the optimisation was terminated, when the iteration on iteration improvement was less than $1 \%$. The optimal vales for $\theta_{n}$ at the range of average channel SNRs are given in Table 2 for the speech-optimised scheme, while for the computer optimised schemes in Table 3.

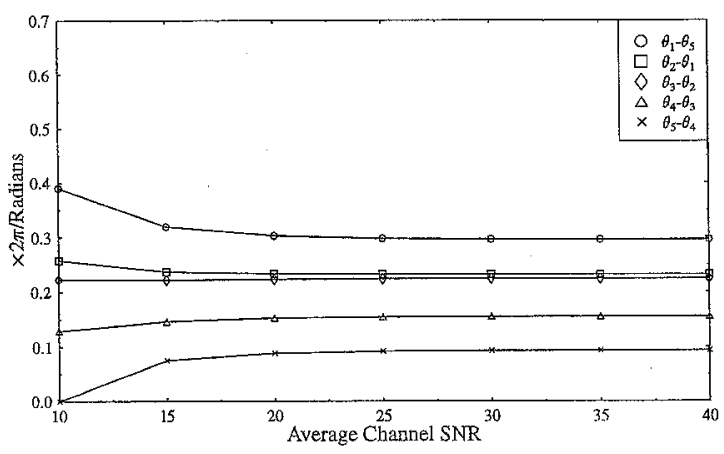

Figure 4: Five PSK decision boundaries with SER optimised for transmission of control symbols of the adaptive modulation speech system in a Rayleigh channel calculated at average SNRs of $10-40 \mathrm{~dB}$

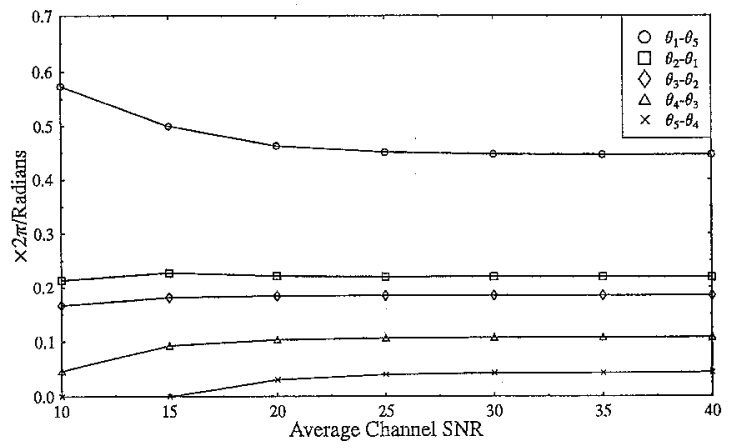

Figure 5: Five PSK decision boundaries with SER optimised for transmission of control symbols of the adaptive modulation computer data system in a Rayleigh channel calculated at average SNRs of 10-40 dB

\section{RESULTS AND CONCLUSIONS}

The results of the phase optimisation are also plotted in Figures 4 and 5 in terms of the optimum phase rotations versus average channel SNR. Both these Figures show clearly that the control symbol transmitted at high SNRs require smaller 'protection zones' compared with the symbols transmitted at lower SNR. In the case of the speech system characterised by Figure 4, it can be seen that the rotation between adjacent phasor positions is less than for the computer data system, while the disabled region's protection zone is wider. Since there is less less protection zone between the speech system's decision angles, there is a reduced overall benefit from employing an un-equal protection control system. Conveniently, however, this is less of a problem, because the acceptable BER in the speech system is higher and therefore control errors are also more acceptable.

Figures 4 and 5 also show that once the average channel SNR exceeds about $25 \mathrm{~dB}$, there is little change in the optimum symbols decision thresholds angles. Therefore, there is little benefit in changing the symbols decision threshold angles on the basis of the long term signal level. Results are shown in Figure 6 for the simulated and numerical solution of the SER using the symbols decision threshold angles, 


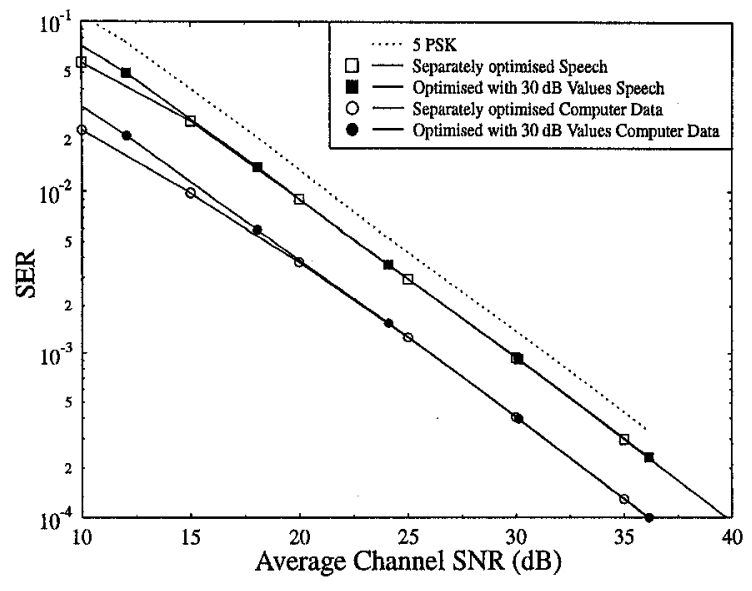

Figure 6: SER through a Rayleigh channel for both speech and computer data optimised systems, for specific average channel SNRs and using the $30 \mathrm{~dB}$ optimised values for all SNRs. The markers represent the simulated and lines the numerical results; 5 PSK numerical results are shown for comparison.

which were optimum at $30 \mathrm{~dB}$. It can be seen that the penalty for using the $30 \mathrm{~dB}$ optimised angles for all SNRs is small, limited to $1 \mathrm{~dB}$ and $2 \mathrm{~dB}$ for the speech and computer data systems, respectively. However, the total SNR benefit of using un-equal protection PSK is in access of 5 $\mathrm{dB}$ for the computer data system and $1 \mathrm{~dB}$ for the speech system. This is virtually a 'zero-cost' improvement and sets an upper bound on the performance for a single control symbol. Multi-symbol performance can easily be determined by exploiting Gfeller's [8] expression.

\section{ACKNOWLEDGEMENT}

The financial support of the EPSRC, UK in the framework of the contract GR/K74043 is gratefully acknowledged.

\section{REFERENCES}

[1] R. Steele and W.T. Webb, "Variable rate QAM for data transmission over rayleigh fading channels," in Wireless '91, Calgary, Alberta, pp. 1-14, IEEE, 1991.

[2] J. G. Proakis, Digital Communications. McGraw-Hill, 1989.

[3] W. H. Press, S. A. Teukolsky, W. T. Vetterling and B. P. Flannery, Numerical Recipes in C. Cambridge University Press, 1994.

[4] J. M. Torrance and L. Hanzo, "Upper bound performance of adaptive modulation in a slow rayleigh fading channel." Electronics Letters, April 1996. pp 169-171.

[5] J. M. Torrance and L. Hanzo, "Optimisation of switching levels for adaptive modulation in a slow rayleigh fading channel." Electronics Letters, June 1996. pp 1167 1169.

[6] S. Otsuki, S. Sampei and N. Morinaga, "Square-QAM adaptive modulation TDMA/TDD systems using modu- lation level estimation with walsh function." Electronics Letters, November 1995. pp 169-171.

[7] K. G. Beauchamp, Walsh functions and their applications. Academic Press, 1975.

[8] Fritz Gfeller, Walter Hirt, Martin de Lange and Beat Weiss, "Wireless infrared transmission: How to reach all office space," in $46^{\text {th }}$ Vehicular Technology Conference, pp. 1535-1539, IEEE, 1996. 